\title{
Stability constraints in modeling of multi-planet extrasolar systems
}

\author{
Krzysztof Goździewski, Cezary Migaszewski and Arek Musieliński \\ Toruń Centre for Astronomy, \\ N. Copernicus University, PL-87-100 Toruń, Poland \\ email: \{k.gozdziewski,c.migaszewski,a.musielinski\}@astri.umk.pl
}

\begin{abstract}
We present an analysis of high precision radial velocity (RV) observations of stars hosting multi-planet systems with Jovian companions. We use dynamical stability constraints and quasi-global methods of optimization. As an illustration, we present new results derived for the RV data of the Sun-like dwarfs HD 155358 and $\tau^{1}$ Gruis.
\end{abstract}

Keywords. radial velocity technique, $N$-body problem, stars: HD 155358, stars: $\tau^{1}$ Gruis

\section{Introduction}

Extrasolar planetary systems have became a major challenge for contemporary astrophysics and dynamical astronomy. One of the most difficult problems in this field concerns the orbital stability of such systems, in particular when related to the observations and their interpretation. Usually, the investigations of long-term evolution are the domain of direct, numerical integrations. The stability of planetary systems is often understood in terms of the Lagrange definition implying that orbits remain well bounded over infinite time. Other definitions may be formulated as well, like the astronomical stability (Lissauer 1999) requiring that the system persists over a very long, Gyr time-scale or Hill stability (Szebehely 1984) that requires the constant ordering of the planets. In our studies, we prefer a more formal and stringent definition related to the fundamental Kolmogorov-Arnold-Moser Theorem (KAM), see Arnold (1978). Planetary N-body systems, involving a dominant mass of the parent star and significantly smaller planetary masses, are well modeled by close-to-integrable, Hamiltonian dynamical systems. According to the KAM Theorem, their evolution may be quasi-periodic (with a discrete number of fundamental frequencies, which are stable forever - and also stable with respect to the other notions of stability quoted above), periodic (or resonant; stable or unstable) or chaotic (with a continuous spectrum of frequencies, and unstable). In the last case, initially close phase trajectories diverge exponentially, i.e., their Maximum Lyapunov Characteristic Exponent (MLCE, denoted also by $\sigma$ ) is positive. This understanding of the global structure of the phase space is widely adopted, in particular with regard to the Solar system dynamics (e.g., Wisdom \& Holman 1991; Laskar 1990; Holman \& Murray 1996; Malhotra 1998; Nesvorný \& Morbidelli 1999; Robutel \& Laskar 2001; Murray \& Holman 2001; Michtchenko \& Ferraz-Mello 2001; Lecar et al. 2001; Morbidelli 2002, and references therein.). However, the distinction between regular and chaotic trajectories is a difficult task that, in practice, may be resolved only with numerical methods relying on efficient and accurate integrators of the equations of motion. 


\section{Stability indicators}

To detect chaotic motions in the phase space, many numerical tools are available. Concerning the dynamics of close to integrable Hamiltonian systems, these tools can be roughly divided onto two classes: spectral algorithms that resolve the fundamental frequencies and/or their diffusion rates (Laskar 1993; Šidlichovský \& Nesvorný 1997; Michtchenko \& Ferraz-Mello 2001), and methods based on the divergence rate of initially close phase trajectories, expressed in terms of the Lyapunov exponents (Benettin et al. 1980; Froeschlé 1984). These fast indicators can be correlated with geometrical evolution of orbital osculating elements, like the maximal eccentricity (max $e)$, maximal amplitude of the critical angle of a resonance $(\max \theta)$ or with event time $T_{E}$ (indicating a collision or an ejection of a body from the system). Unfortunately, no general relation between these indicators can be determined (Lecar et al. 2001; Michtchenko \& Ferraz-Mello 2001). The event time $T_{E}$, relying on CPU-intensive long-term integrations of the equations of motion (Lecar et al. 2001) can be considered as a direct measure of the astronomical stability.

In our work, among the the spectral tools, we often choose the method invented by Michtchenko \& Ferraz-Mello (2001); its idea is very simple - to detect chaotic behavior one counts the number of frequencies in the FFT-spectrum of an appropriately chosen dynamical signal. We deal with conservative Hamiltonian systems; so in a regular case, the spectrum of fundamental frequencies is discrete and we obtain only a few dominant peaks in the FFT spectrum. Chaotic signals do not have well defined frequencies, and their FFT spectrum is very complex. The number of peaks in the spectrum above some noise level $p$ (typically, $p$ is set to a few percent of the dominant amplitude) tell us about the character (regular vs chaotic) of a given phase trajectory.

The basic tool to discover exponentially unstable bounded orbits, i.e. chaotic orbits, is the Maximum Lyapunov Characteristic Exponent (MLCE) $\sigma$. The direct computation of the MLCE is based on the analysis of the tangent vectors $\boldsymbol{\delta}$ which are solutions to the variational equations of the equations of planetary motions:

$$
\frac{\mathrm{d}}{\mathrm{d} t} \boldsymbol{x}=\boldsymbol{f}(\boldsymbol{x}),
$$

where $\boldsymbol{x}$ denotes the state vector including coordinates and momenta, and $\boldsymbol{f}$ stands for the gravitational forces. For its solution $\phi=\phi(t)$ we define:

$$
\frac{\mathrm{d}}{\mathrm{d} t} \boldsymbol{\delta}=\boldsymbol{A}(t) \boldsymbol{\delta}, \quad \boldsymbol{A}(t):=\frac{\partial \boldsymbol{f}}{\partial \boldsymbol{x}}[\phi(t)], \quad \delta=\|\boldsymbol{\delta}\| .
$$

Asymptotically, the MLCE value is given by (Cincotta \& Simó 2000):

$$
\sigma=\lim _{t \rightarrow \infty} \frac{1}{t} \int_{0}^{t} \frac{\dot{\delta}(s)}{\delta(s)} \mathrm{d} s .
$$

If $\sigma$ converges to some positive value, we conclude that the nominal orbit $\phi$ and some initially close orbit diverge exponentially at the rate $\exp (\sigma t)$. Two practical difficulties arise when the direct definition (2.2) is used: the convergence of $\sigma$ is often very slow, and it is difficult to tell how small the final value of $\sigma$ should be to consider it $\sigma=0$.

A large variety of methods has been proposed to overcome the problem of slowly convergent MLCE estimates. Recently, a new algorithm offering excellent convergence, called MEGNO (Mean Exponential Growth factor of Nearby Orbits), has been proposed by Cincotta \& Simó (2000). The definition of MEGNO and its mean value is the following 
(Cincotta et al. 2003):

$$
Y(t)=\frac{2}{t} \int_{0}^{t} \frac{\dot{\delta}(s)}{\delta(s)} s \mathrm{~d} s, \quad\langle Y\rangle(t)=\frac{1}{t} \int_{0}^{t} Y(s) \mathrm{d} s .
$$

It was shown that if $\phi(t)$ is a regular solution with a linear divergence of nearby orbits then $\lim _{t \rightarrow \infty}\langle Y\rangle(t)=2$, and if $\phi(t)$ is a chaotic solution then $\langle Y\rangle(t) \sim(\sigma / 2) t$, as $t \rightarrow \infty$. Moreover, when $\langle Y\rangle(t)$ tends towards a value different from 2 , then it indicates that close trajectories diverge according to a certain power law. If $\phi(t)$ is a periodic solution then $\langle Y\rangle(t)$ tends to 0 . The asymptotic behavior of $\langle Y\rangle(t)$ is given by a uniform formula $\langle Y\rangle(t) \sim a t+d$, where $a \sim 0$ and $d \sim 2$ for a quasi-periodic solution, while $a \sim \sigma / 2$ and $d \sim 0$ for an irregular and stochastic motion. Having $Y(t)$ we can indirectly estimate the MLCE on a finite time interval. The weight function $s$ in the definition of MEGNO reduces the contribution of the initial part of the tangent vector evolution, when the exponential divergence is too small to be observed relative to other linear and nonlinear effects (Morbidelli 2002). Thus, fitting the straight line to the final part of $Y(t)$, we obtain good estimates of $\sigma$ from a relatively shorter piece of trajectory than in the direct MLCE evaluation.

\section{Modeling the RV data - an overview}

The radial velocity (RV) is still the most efficient technique for detecting extrasolar planets. To model the RV signal, the standard formulae by Smart (1949) are commonly used. Each planet in the system contributes to the reflex motion of the star at time $t$ with:

$$
V_{\mathrm{r}}(t)=K[\cos (\omega+\nu(t))+e \cos \omega]+V_{0},
$$

where $K$ is the semi-amplitude, $\omega$ is the argument of pericenter, $\nu(t)$ is the true anomaly (involving implicit dependence on the orbital period $P$ and time of periastron passage $\left.T_{\mathrm{p}}\right), e$ is the eccentricity, $V_{0}$ is the velocity offset. We interpret the primary model parameters $\left(K, P, e, \omega, T_{\mathrm{p}}\right)$ in terms of the Keplerian elements and minimal masses related to coordinates of Jacobi (Lee \& Peale 2003) or Poincaré (Ferraz-Mello et al. 2006).

In our previous work, we tested and tried to optimize different tools helpful for exploring the multi-parameter space of $\left(\chi_{\nu}^{2}\right)^{1 / 2}$ for the model Eq. 3.1 and its generalizations. In the case when $\left(\chi_{\nu}^{2}\right)^{1 / 2}$ may possess many local extrema, we found that good results can be obtained with hybrid optimization (Goździewski \& Migaszewski 2006). A single run of the hybrid code starts the quasi-global genetic algorithm (GA, Charbonneau 1995). GA makes it easy to carry out a constrained optimization within prescribed parameter bounds or to add a penalty term to $\left(\chi_{\nu}^{2}\right)^{1 / 2}$. The best fits found with GAs are not very accurate in terms of $\left(\chi_{\nu}^{2}\right)^{1 / 2}$, so finally, a number of the best fit members of the "population" are refined using a relatively fast local method like the simplex of Melder and Nead (Press et al. 1992). The simplex is a matter of choice, so we could use other fast local methods. However, the code using non-gradient methods works with minimal requirements for user-supplied information. It is only required to define the model function ]the so called fitness function, usually equal to $\left.1 /\left(\chi_{\nu}^{2}\right)^{1 / 2}\right]$ - conveniently, this function is the same for the GAs and simplex - and to determine (even very roughly) the bounds of the parameters. The repeated runs provide an ensemble of the best-fits that helps us to detect local minima of $\left(\chi_{\nu}^{2}\right)^{1 / 2}$, even if they are distant in the parameter space. We can also obtain reliable approximation to the parameter errors (Bevington \& Robinson 2003) within the $1 \sigma, 2 \sigma$ and $3 \sigma$ confidence intervals of $\chi^{2}$ at selected 2-dim parameter planes. 
While we prefer the GAs as the quasi-global optimization tool, other efficient and robust algorithms for identifying and characterizing multiple planetary orbits in precision RV data are known. In particular, the Bayesian Kepler periodogram (Gregory 2007) and Markov chain Monte Carlo (MCMC) technique (Ford 2005) are proven to be robust tools for calculating the model marginal likelihood which is used to compare the probabilities of models with different numbers of planets and for investigating the uncertainty of parameters in the orbital solutions.

Due to the limited time-span of the observations, we often encounter a problem that the data only partially cover the longest orbital period. In this situation, it is possible either that $\left(\chi_{\nu}^{2}\right)^{1 / 2}$ does not have a well defined minimum, or that its shape is very "flat", so the confidence levels may cover large ranges of the fit parameters. To illustrate the shape of $\left(\chi_{\nu}^{2}\right)^{1 / 2}$ in selected 2-dim parameter planes, we perform a systematic scanning of the space of initial conditions with the fast Levenberg-Marquardt (L-M) algorithm (Press et al. 1992). Usually, for representing such scans, we choose the semimajor-axiseccentricity $(a, e)$ plane of the outermost planet. We fix $(a, e)$ and then search for the best fit, initiating the L-M algorithm with starting points selected randomly (but within reasonably wide parameter bounds). The L-M scheme ensures a rapid convergence. It is heavily CPU-consuming and may be effectively applied in low-dimensional problems. In reward it provides a clear picture of the parameter space.

In the case of resonant or mutually interacting planets, the problem is even more complex. How to interpret the RV measurements in that case often remains an open and difficult question. The $N$-planet configurations are parameterized by at least $5 N+$ 1 parameters, even assuming that the system is coplanar. The RV signal in terms of Eq. 3.1 is degenerate - we have no information on the inclinations of orbits and the true masses of the companions. Moreover, we do not know a priori the number of planets in the system, so the resolved solutions are often not unique. It is also well known that the Keplerian (kinematic) model is usually not adequate to properly explain the RV variability. Instead, a self-consistent $N$-body Newtonian model should be applied (Rivera \& Lissauer 2001; Laughlin \& Chambers 2001). Basically, the effects of mutual interactions included in the Newtonian model could make it possible to determine or estimate the inclinations and masses provided that long enough time-series of precision data are available. Nevertheless, the measurements can be affected by many sources of error, like complex systematic instrumental effects, short time-series of the observations, irregular sampling due to observing conditions, and stellar noise. Little is known on the real statistical characteristics of the error distributions and the assumption that these are Gaussian distributions is not necessarily valid (Ferraz-Mello et al. 2005), see also (Baluev 2007).

All these factors, in particular the unspecified number of planets and undetermined or weakly constrained parameters, can (and often do) lead to best-fit solutions representing unrealistic, quickly disrupting configurations (Ferraz-Mello et al. 2005; Lee et al. 2006; Goździewski \& Konacki 2006). But according to the Copernican principle, the detection of strongly unstable systems during two decades of RV observations is not likely, we would rather expect that the dynamical stability should be preserved over a significant part of the parent star life-time counted in Gyrs. Stability is therefore a natural requirement of a model solution consistent with observations. Many authors take it into account when analyzing the dynamics of the best-fit configurations with different constraints, e.g., to mention only a few examples in an endless list of references: through long-term integrations, requiring astronomical stability (Lissauer \& Rivera 2001; Laughlin et al. 2002) or Lagrange and/or Hill stability (Barnes \& Greenberg 2007), also through fast indicators like the diffusion of characteristic frequencies (Correia et al. 2005), fast Lyapunov 
indicators (Dvorak et al. 2005), maximal eccentricity (Vogt et al. 2005; Ford et al. 2005), critical angles ( $\mathrm{Ji}$ et al. 2003; Lee et al. 2006; Beaugé et al. 2006, see also paper by Beaugé et al. (2008) in this volume), or $T_{E}$ determined over short-time scale (the Systemic project initiated and led by Greg Laughlin, www.oklo.org). However, a common approach to search for stable solutions in a neighborhood of unstable best-fit configuration by trial and error does not necessarily provide stable fits that are simultaneously optimal, in terms of $\left(\chi_{\nu}^{2}\right)^{1 / 2}$ or an rms. What is even more important, the stability requirement imply non-continuous and complex structure of the space of initial conditions that depends on adopted definition of stability.

Hence, as a general way of modeling the observations we propose to eliminate unstable (for instance, strongly chaotic) solutions during the fitting procedure. The idea is very simple: we modify the hybrid algorithm by adding a suitable penalty term to the function determining the fit quality, e.g., $\left(\chi_{\nu}^{2}\right)^{1 / 2}$ or rms for unstable solutions. The penalty term must rely on some signature of the system stability. We found that MEGNO is particularly useful for that purpose thanks to its rapid convergence and great sensitivity to chaotic motions. We have shown that on many examples, this method (called Genetic Algorithm with MEGNO Penalty, GAMP) is very useful in modeling resonant or closeto-resonant planetary configurations when even small errors of orbital phases or other parameters may lead to quick self-destruction of the system. Unfortunately, the algorithm cannot give a definite answer when we want to resolve the $\left(\chi_{\nu}^{2}\right)^{1 / 2}$ shape in detail or find strictly stable solutions because, in practice, the penalty term can be calculated only a over relatively short period of time (due to CPU time requirements). This is particularly important for systems affected by long-term resonances, i.e., configurations with three and more planets, inclined orbits (Goździewski et al. 2007). Hence, in an additional step, we need to examine the stability of individual best fits selected with GAMP over a period of time related to the time-scale of relevant unstable behaviors (resonances).

\section{HD 155358 - a system with two planets}

To illustrate the algorithms and problems discussed above on a new planetary system, we consider the RV observations of HD 155358 by Cochran et al. (2007). The data published in this paper consist of 71 observations and span $\sim 2100$ days. The singleplanet Keplerian model does not fully explain the RV variability, so the discovery team studied a 2-planet Keplerian model of the RV. This yields three acceptable fits: a solution which is stable for 100 Myrs with $P_{\mathrm{c}} \sim 195$ days, $P_{\mathrm{c}} \sim 526$ days and minimizes $\left(\chi_{\nu}^{2}\right)^{1 / 2}$ as well as two unstable fits with $P_{\mathrm{c}} \sim 500$ and 1500 days, respectively. These fits have very large $e_{\mathrm{c}}$ leading to catastrophically unstable configurations (Cochran et al. 2007).

We try to extend the analysis of the RV data by considering an $N$-body model of the observations and looking more closely at the phase-space structure of the putative 2-planet system with the help of the dynamical tools described above.

At first, we did two systematic scans of $\left(\chi_{\nu}^{2}\right)^{1 / 2}$ in the $\left(P_{\mathrm{c}}, e_{\mathrm{c}}\right)$-plane. The results are shown in Fig. 1. Three local minima of $\left(\chi_{\nu}^{2}\right)^{1 / 2}$ reported by Cochran et al. $(2007)$ are evident. Two of them lie far over the collision line of orbits, defined in terms of semiaxes and eccentricities through $a_{b}\left(1+e_{b}\right)=a_{c}\left(1-e_{c}\right)$. This line marks the zone in which the mutual interactions of relatively massive companions can quickly destabilize the configuration. The solution with $P_{\mathrm{C}} \sim 300$ days yields $\left(\chi_{\nu}^{2}\right)^{1 / 2}$ similar to that one of the best fit with $P_{\mathrm{c}} \sim 500$ days.

Next, we refined the Keplerian fits with $N$-body model. The osculating parameters of the dominant solution (see Table 1, fit II) are slightly different from that one of the Kepler model, nevertheless it appears also stable. Its neighborhood is illustrated in dynamical 

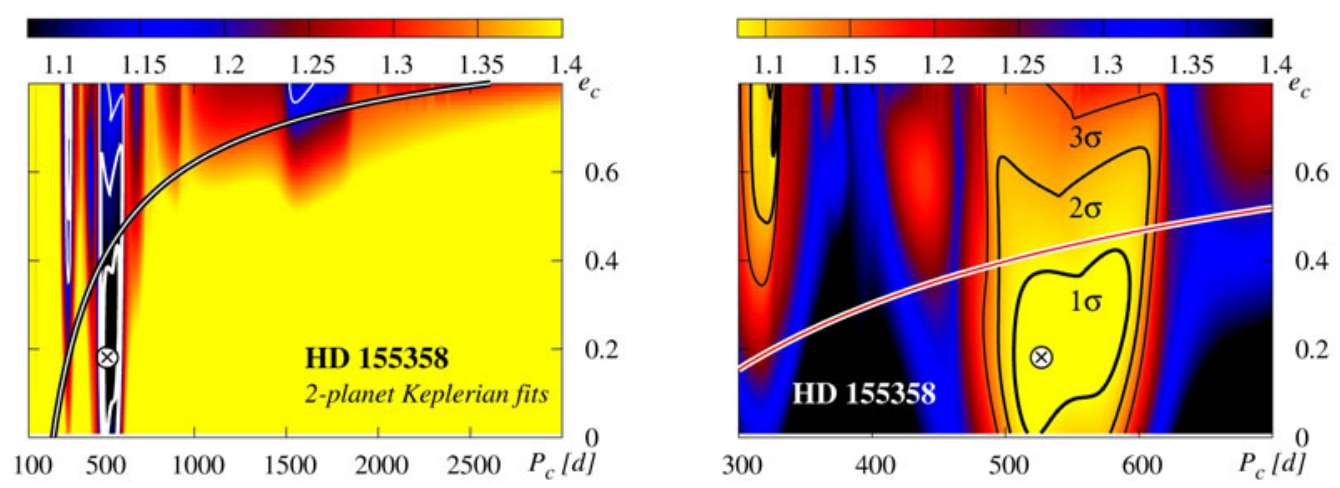

Figure 1. The levels of reduced $\left(\chi_{\nu}^{2}\right)^{1 / 2}$ of the 2-planet Keplerian model of the HD 155358 RV data published in Cochran et al. $(2007)$ onto the $\left(P_{\mathrm{c}}, e_{\mathrm{c}}\right)$-plane. The left panel is for the whole tested range of parameters. The right panel is for the close-up of the most prominent minima around $P_{\mathrm{C}} \sim 500$ days and $e_{\mathrm{C}} \sim 0.2$. The smooth line is for the collision line. Curves labeled with $1 \sigma, 2 \sigma$ and $3 \sigma$ are for the confidence levels of the best fit marked with crossed circle.

maps shown in Fig. 2. Curiously, the best-fit configuration it located in the very edge of a stable zone between 5:2 and 3:1 mean motion resonances (MMRs) of two planets with masses $\sim 0.8 \mathrm{~m}_{\mathrm{J}}$ and $\sim 0.5 \mathrm{~m}_{\mathrm{J}}$, respectively. As we have observed in other cases, the $\max e$ and $\max \theta$ indicators are in excellent correlation with the measure of formal stability (here, $\log S N$ ). We can also see a very complex border of the stable zone. The system would be located in dynamically active region of the phase space spanned by a few low-order MMRs. The proximity of the best fit configuration to the 5:2 MMR and moderate eccentricities indicate a dynamical similarity of the HD 155358 system to the Solar system.

To illustrate the hybrid optimization, we performed an independent search for 2-planet Keplerian fits assuming orbital periods in the range of [100,3000] days and eccentricities in the range of $[0,0.8]$. The ensemble of gathered fits is shown in the top-left panel in Fig. 3 . To make the comparison with the results of systematic scanning more transparent, in this panel we also plot contour levels of $1 \sigma, 2 \sigma$ and $3 \sigma$ confidence intervals seen in Fig. 1 . Note that we plot only solutions in the range of $P_{\mathrm{c}} \in[300,700]$ days (compare with the right panel in Fig. 1). The hybrid algorithm also reveals the minima seen in Fig. 1. Besides, it detected one more minimum at $P_{\mathrm{c}} \sim 300$ days and moderate $e_{\mathrm{c}} \sim 0.1$, at the same depth. This justifies the efficiency and robustness of the hybrid code. The algorithm not only detects the best fits solutions but also helps to resolve to some extent the shape of $\left(\chi_{\nu}^{2}\right)^{1 / 2}$.

The best fits with $P_{\mathrm{c}} \sim 300$ days are both very unstable. It does not necessarily mean that in their neighborhood some stable solutions do not exist, so it is the case in which the application of GAMP can be helpful. Indeed, we can detect two clumps of stable fits (see the top-right panel of Fig. 3) in the regime of large $e_{\mathrm{c}}$, both lying far over the collision line. In one of these islands, we pick up a rigorously stable solution with $\left(\chi_{\nu}^{2}\right)^{1 / 2} \sim 1.14$ comparable to that one of the best fit II (Table 1) yielding only marginally worse rms $\sim 6.3 \mathrm{~m} / \mathrm{s}$. The evolution of MEGNO and osculating elements in this quasi-periodic configuration is shown in Fig. 4. We notice extremely large variations of eccentricities up to 0.8 . The system would be involved in 5:3 MMR protecting companions from close 

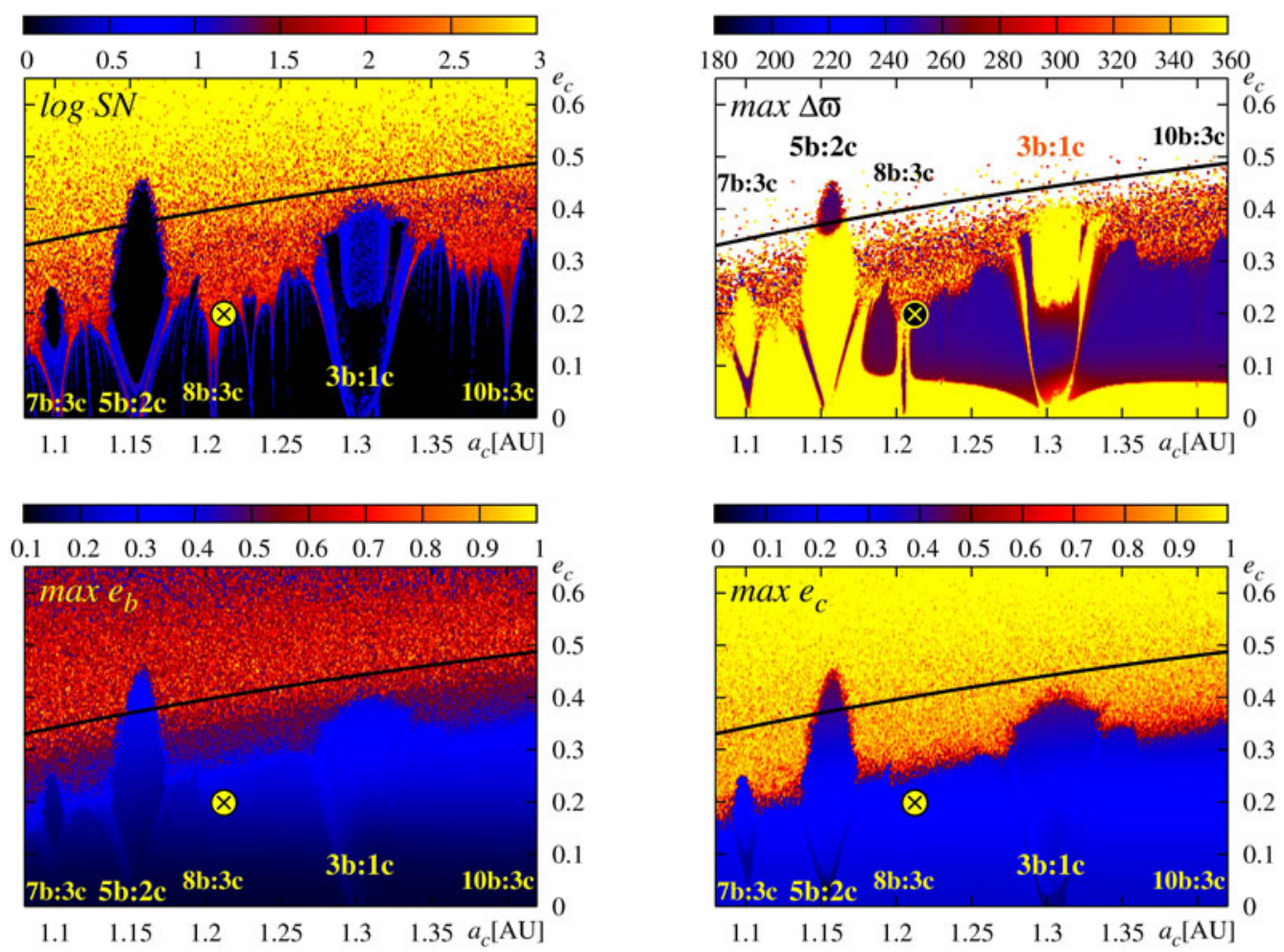

Figure 2. Dynamical maps of putative HD 155358 coplanar configuration of two Jovian planets $\left(\sim 0.5-0.9 \mathrm{~m}_{\mathrm{J}}\right)$. The osculating elements of the $N$-body solution at the epoch of the first observation in Cochran et al. (2007) are given in Table 1 (fit II) and marked with crossed circle. The top-left panel is for the Spectral Number. Colors mark the stability regime: black is for regular solutions, yellow is for strongly chaotic solutions. The top-right panel is for the maximal amplitude of apsidal angle $\Delta \varpi=\varpi_{\mathrm{c}}-\varpi_{\mathrm{b}}$. Panels in the bottom row are for the max $e$ indicator (i.e., the maximal eccentricity attained after the integration period $\sim 30,000 \mathrm{yr}$ ). The most prominent mean motion resonances between the planets are labeled.

encounter (see evolution of the critical argument of this resonance in the bottom-right panel in Fig. 4).

Yet the fit parameters are determined within some error ranges that should be interpreted with taking into account the structure of the phase space (see Fig. 2). To illustrate this problem we examined more closely the neighborhood of the best fit II. At this time, we performed two experiments. In the first search, we applied the hybrid code without stability constraints driven by the "usual" $N$-body model of the RV. The results are illustrated in the bottom-left panel in Fig. 3. The quality of fits within $1 \sigma, 2 \sigma$ and $3 \sigma$ confidence intervals of the best fit (marked with crossed circle; see Table 1, fit II) is color coded with blue, light-blue and gray, respectively. The best fits only marginally worse from the best one, are marked in red. Curiously, the plot reveals a subtle structure with three additional local minima of $\left(\chi_{\nu}^{2}\right)^{1 / 2}$, in relatively small range of $a_{\mathrm{c}} \in[1.1,1.3] \mathrm{AU}$. Moreover, these minima are spread over wide range of $e_{\mathrm{c}} \in[0,0.7]$. Simultaneously, this zone covers many low order resonances, between 5:2 and 3:1 MMR and the $\left(\chi_{\nu}^{2}\right)^{1 / 2}$ "valley" is crossed by the collision line. Close to this line, the stability could be preserved only if the planets are protected from close encounter through an MMR. 

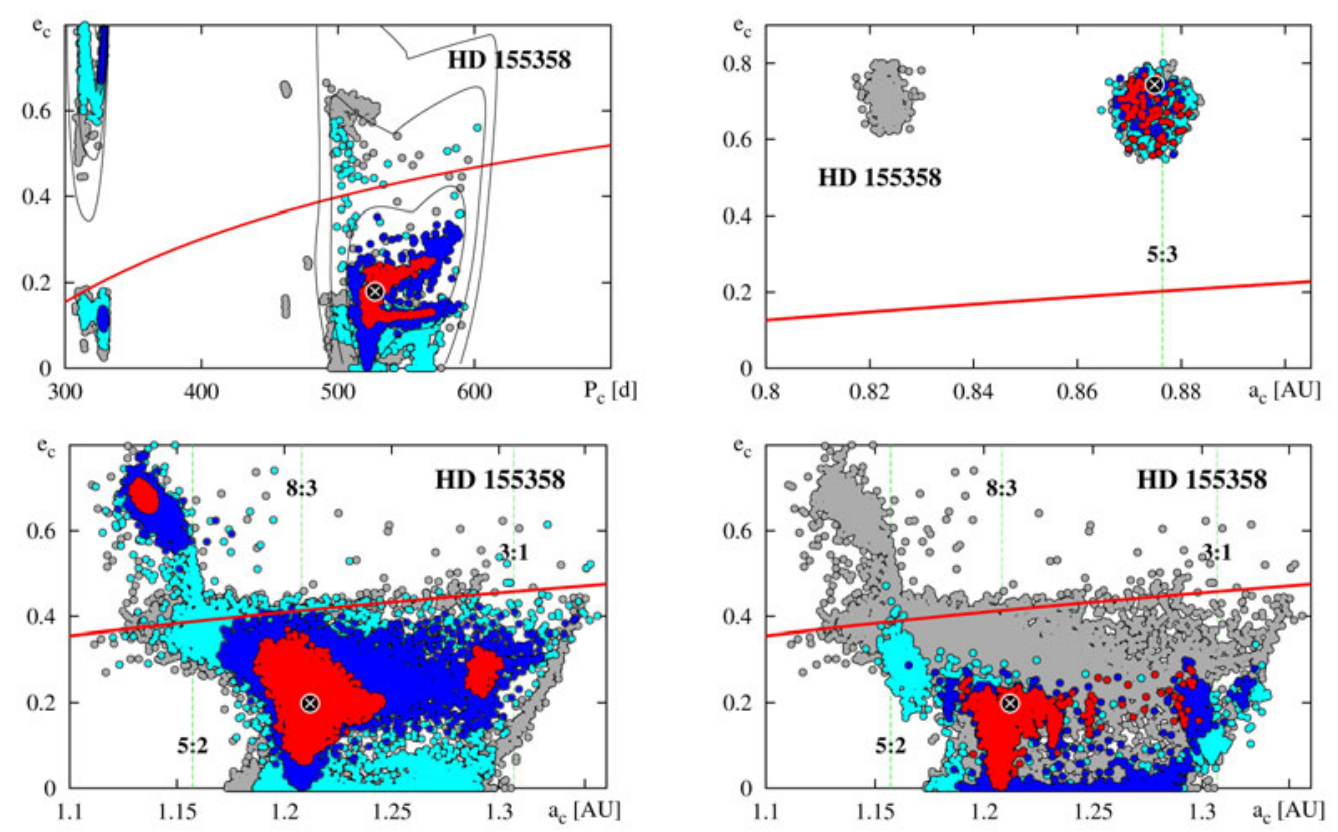

Figure 3. The top-left panel is for the $\left(\chi_{\nu}^{2}\right)^{1 / 2}$ of the 2-planet Keplerian solutions to the HD 155358 RV data published in Cochran et al. (2007) derived with the hybrid algorithm. The fit parameters are projected onto the $\left(P_{\mathrm{c}}, e_{\mathrm{c}}\right)$-plane. Their quality is color coded: dark blue is for $1 \sigma$-, light-blue is for $2 \sigma$ - and grey is for $3 \sigma$-confidence levels of the best fit marked with crossed circle. Contours are for the confidence levels obtained in the systematic scan (see the right panel in Fig. 1). The top-right panel is for stable solutions in the range $P_{\mathrm{C}} \sim 300$ days derived with GAMP driven by the $N$-body model. The stable best-fit solution in this area is marked with crossed circle, and its elements are given in Table 1, fit I. The bottom-left panel is for the ensemble of fits gathered with the hybrid algorithm driven by the $N$-body model of the RV data around the dominant minimum of $\left(\chi_{\nu}^{2}\right)^{1 / 2}$. The bottom-right panel illustrates stable solutions gathered in the GAMP search. The set of solutions within $3 \sigma$ of the best stable fit (elements given in Table 1, fit II) but obtained without stability constraints, as shown in the bottom-left panel, are again shown as a gray-filled contour. Approximate positions of the most prominent MMRs are labeled.

Now, we could examine the stability of every fit that we found but we choose a new search for stable solutions in a self-consistent manner with GAMP. The results are shown in the bottom-right panel in Fig. 3. In this panel, we overplot the stable solutions within $1 \sigma, 2 \sigma$ and $3 \sigma$ confidence interval of the best stable fit II over all solutions within $3 \sigma$ level which are found in the previous search (i.e., without stability constraints). It now is evident that only a part of the $\left(\chi_{\nu}^{2}\right)^{1 / 2}$ valley can consist of dynamically stable solutions, nevertheless the acceptable fits are spread over significant range of $\Delta a_{\mathrm{c}} \sim 0.2 \mathrm{AU}$. Apparently, this error is quite small but in fact it is large enough to cover a few low-order MMRs. We conclude that the current set of RV data cannot fully characterize the system state and new observations are required to constrain the elements of the outer planet.

Finally, both Keplerian and Newtonian 2-planet solutions lead to apparent excess of the residuals, in particular at the end parts of the RV curve. It may indicate that the 2-planet model does not fully explain the RV variability. In particular, the system may involve more than two planets. A heuristic argument supporting such a claim may be the proximity of the best fits to the collision line. We know similar cases, for instance $\mu$ Arae (Goździewski et al. 2007; Pepe et al. 2007), or HD 37124 (Vogt et al. 2005). To 

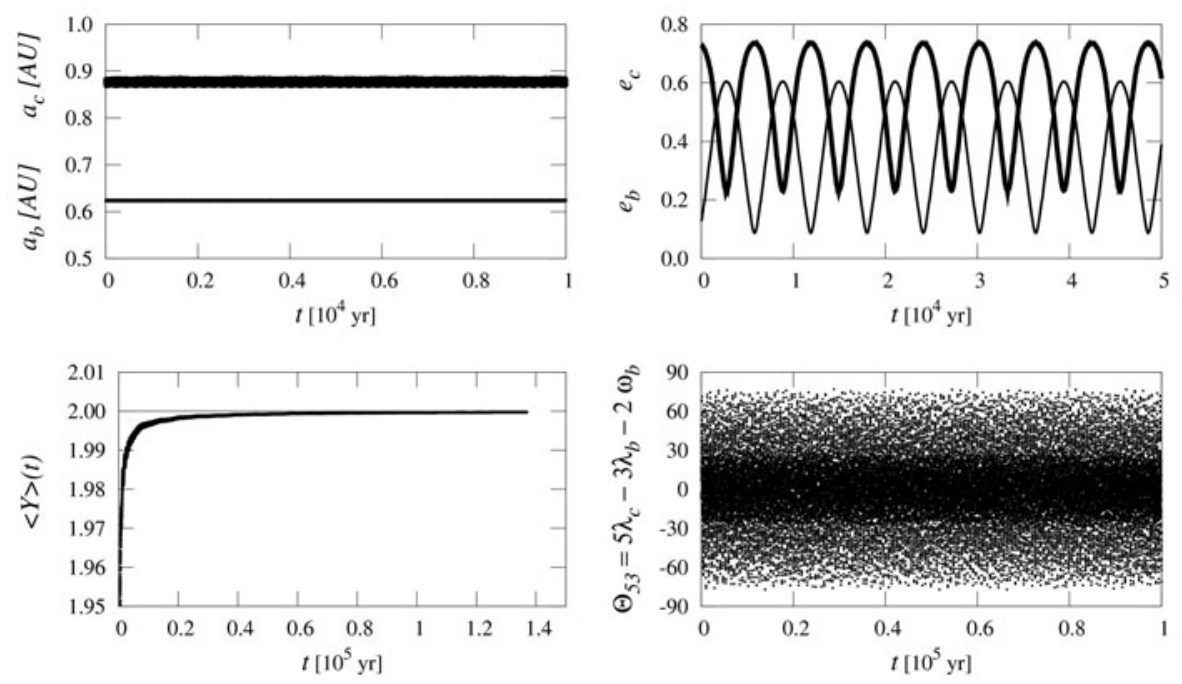

Figure 4. The temporal evolution of orbital elements in the 2-planet, Newtonian solution to the RV data of HD 155358 related to highly eccentric orbits (see the top-left panel in Fig. 3). The osculating elements are given in Table 1 (fit I). The top-left panel is for the semi-major axes, the top-right panel is for the eccentricities. The MEGNO is plotted in the bottom-left panel. The bottom-right panel is for the critical angle of the 5:3 MMR.

check such hypothesis we looked first for 3-planet Keplerian solutions with the hybrid code. The best fit found yields $\left(\chi_{\nu}^{2}\right)^{1 / 2} \sim 0.87$ and significantly better $\mathrm{rms} \sim 4.6 \mathrm{~m} / \mathrm{s}$ but is unstable. The GAMP search yields stable configurations with quasi-circular orbits of the outermost planets, yielding $\mathrm{rms} \sim 5.5 \mathrm{~m} / \mathrm{s}$. An example fit of this type, yielding $\left(\chi_{\nu}^{2}\right)^{1 / 2} \sim 1.07$ and an $\mathrm{rms} \sim 5.6 \mathrm{~m} / \mathrm{s}$, given in terms of osculating element at the epoch of the first observation in tuples of ( $\left.m\left[\mathrm{~m}_{\mathrm{J}}\right], a[\mathrm{AU}], e, \omega[\mathrm{deg}], \mathcal{M}\left(t_{0}\right)[\mathrm{deg}]\right)$ is the following $(0.115,0.383,0.025,281.3,154.4),(0.770,0.627,0.040,108.9,173.7),(0.490$, $1.187,0.000,359.1,65.7)$, for planets $d, b, c$ respectively, the offset $V_{0}=10.21 \mathrm{~m} / \mathrm{s}$. We note the small mass of the innermost planet. Its RV signal is at the level of noise, so additional observations would be required to confirm of withdrawn such a model.

\section{Trojan planets in the $\tau^{1}$ Gruis system?}

Laughlin \& Chambers (2002) predict that the reflex signal of a single planet in a quasi-circular orbit may be also interpreted by two Jovian Trojan planets, i.e., two objects sharing similar orbits (involved in 1:1 MMR). That possibility is intriguing because stable Trojan companions to the stars may be quite common. It can be indicated by a number of stable Trojan configurations in the Solar system. Some argue that they can be a frequent by-product of planet formation and and/or dynamical evolution (Laughlin \& Chambers 2002). However, the genesis of Trojan planets is not quite clear because on the contrary, there is some evidence that formation of such bodies could be difficult (Beaugé et al. 2007). Still, many authors expect that Trojan planets can exist [see, for instance, the work of Dvorak et al. in this volume and references therein, also (Ford \& Gaudi 2006)].

Recently, we found a similar kind of ambiguity of the RV models concerning 2:1 MMR configurations. At present, we know five extrasolar systems presumably involved in 
2:1 MMR, i.e., Gliese 876 (Marcy et al. 2001), HD 82943 (Mayor et al. 2003), HD 128311 (Vogt et al. 2005), HD 73526 (Tinney et al. 2006), and $\mu$ Arae (Jones et al. 2003; Goździewski et al. 2007; Pepe et al. 2007). However, the 2:1 MMR model of the radial velocity observations can also be non-unique. The periodogram of the 2:1 MMR RV signal is very similar to that one of the 1:1 MMR. Indeed, the RV variability of HD 128311 and HD 82943 can be explained by highly inclined systems in 1:1 MMR (Goździewski \& Konacki 2006). We also found that the RV of HD 73526 can be modeled with two highly inclined Jovian Trojans. The modeling of the 1:1 MMR is a challenging problem because Jupiter-like planets sharing eccentric orbits with similar semi-major axes interact heavily and the collisional configurations are generic. Hence, stability constraints are critical in the search for optimal and stable configurations. This seems to be one of the best applications of GAMP like algorithms.

Among a few cases we analyzed so far, the $\tau^{1}$ Gruis appears to be a particularly interesting example of the possible "Jupiter on circular orbit"- "two Trojans" ambiguity. A Jovian companion to the G0 dwarf $\tau^{1}$ Gruis in a wide and almost circular orbit has been announced in the work by Jones et al. (2003). In our analysis, we use updated RV data comprising of 59 precision measurements (Butler et al. 2006). The best-fit single planet model to these data yields $P_{\mathrm{b}} \sim 1300$ days, and $e_{\mathrm{b}} \sim 0.1$. We re-analyse the data to look for possible Trojan planet solutions. Curiously, we quite easily found many stable, coplanar configurations involved in 1:1 MMR (Fig. 5) yielding similar or slightly better fit quality $(\mathrm{rms} \in[5,6] \mathrm{m} / \mathrm{s}$ ). The reflex signal of the 1:1 MMR (Fig. 6) can hardly be distinguished from that of a single-planet system. The osculating elements of the Trojans are given in Table 1 (fit III). In this case, both planets would move on quasi-circular orbits and these would be coplanar. The dynamical maps shown in Fig. 5 (also accompanying other best-fits solutions with acceptable quality which we found in the search) illustrate the extreme variability of the 1:1 MMR islands. The map for the best-fit with elements in Table 1 is shown in the top-left panel of Fig. 5. Another peculiar solution is illustrated in the top-right map in Fig. 5. The initial eccentricities are moderate, and the system would be found in extremely large island of stable motions. It spans whole range of $e_{\mathrm{b}}$. The possibility of existence of such extended stable zones may strength the hypothesis of stable extrasolar Trojans.

The ambiguity of the RV fits implies interesting issues concerning the models of creation and stability of Earth-like planets interior to the orbits of the putative Jovian Trojans. In the $\tau^{1}$ Gruis, the space interior to the Jovian planet is "empty" as no smaller planets have been yet detected. So we can try to predict in which regions of the habitable zone $(\sim 1 \mathrm{AU})$ smaller planet could survive. For this purpose we computed dynamical maps for putative Earth-like masses with initial conditions varied in the $\left(a_{0}, e_{0}\right)$ plane, and initial orbital angles set to $0^{\circ}$. We considered two dynamical environments: the one with the best-fit Jovian companion in close to circular orbit and the second one with Trojans in quasi-circular orbits (their elements are given in Table 1, fit III). The results are shown in Fig.7. For the first configuration, we detect an extended zone of stable motions. Additional experiments regarding creation of Earth-like planets through coagulation of Mars and Moon-size protopolanets (see Raymond, 2008 in this volume) performed with the Mercury code (Chambers 1999) assures us that such planets emerge easily in that zone. In the case of a configuration with Trojans, the stable zone shrinks significantly. Moreover, the creation of Earth-like planets is much more difficult. We found that they could form only in the zones of relatively stable motions, up to $\sim 0.9 \mathrm{AU}$ and in the "gap" between the 4:1 MMR and the border of global instability. Yet in that case, the simulations are very difficult to carry out due to frequent close encounters between planetesimals and the Jovian planets. 

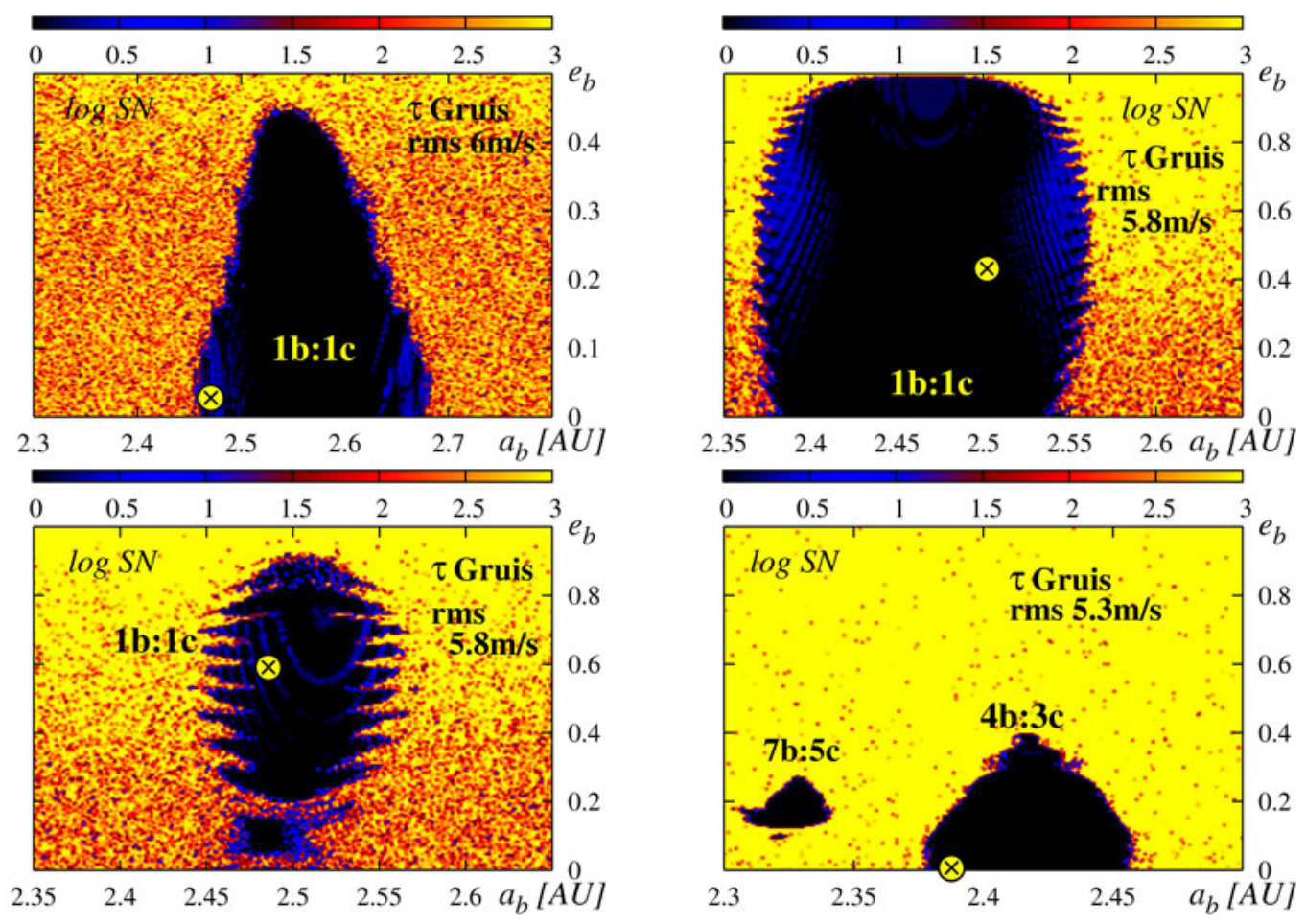

Figure 5. Dynamical maps of putative $\tau^{1}$ Gruis coplanar, edge-on configuration of two Jovian planets $\left(\sim 0.5-0.9 \mathrm{~m}_{\mathrm{J}}\right)$ involved in low-order resonances. The best-fits yield an $\mathrm{rms} \sim 5-6 \mathrm{~m} / \mathrm{s}$ and $\left(\chi_{\nu}^{2}\right)^{1 / 2} \sim 1$. Their quality is similar to that of the single-planet solution (an rms about of $6 \mathrm{~m} / \mathrm{s})$.

\section{Conclusions}

In this work we consider some problems related to modeling observations of stars hosting multi-planet systems. It is well known that the phase space of such system has a non-continuous and complex structure with respect to any stability criterion. Hence, when searching for initial conditions, one has to take into account the dynamical character of putative planetary configurations. Due to narrow observational windows, significant measurement errors, stellar jitter and other uncertainties, the formal best-fits may appear very unstable. Searching for stable solutions in their neighborhood of the phase space by trial and error, we should not expect that the results could be statistically optimal. Thus, an intuitively natural approach is to eliminate unstable configurations during the fitting process, through penalizing unstable solutions with a suitably large value of the $\left(\chi_{\nu}^{2}\right)^{1 / 2}$ function, or of another measure of the fit quality. In that way, the stability plays a role of an additional, implicit observable. That method is suitable for multi-body systems with Jovian planets presumably involved in low-order mean motion resonances (MMRs). In particular, we considered two new examples in which the model of the RV may be non-unique. The RV of HD 155358 by Cochran et al. (2007) permit a few local minima of $\left(\chi_{\nu}^{2}\right)^{1 / 2}$ related to different orbital configurations. We also found an example illustrating the ambiguity of Keplerian, close to circular single-planet solutions. The RV data of $\tau^{1}$ Gruis could be equally well modeled with coplanar configurations of Jovian planets involved in 1:1 MMRs. 


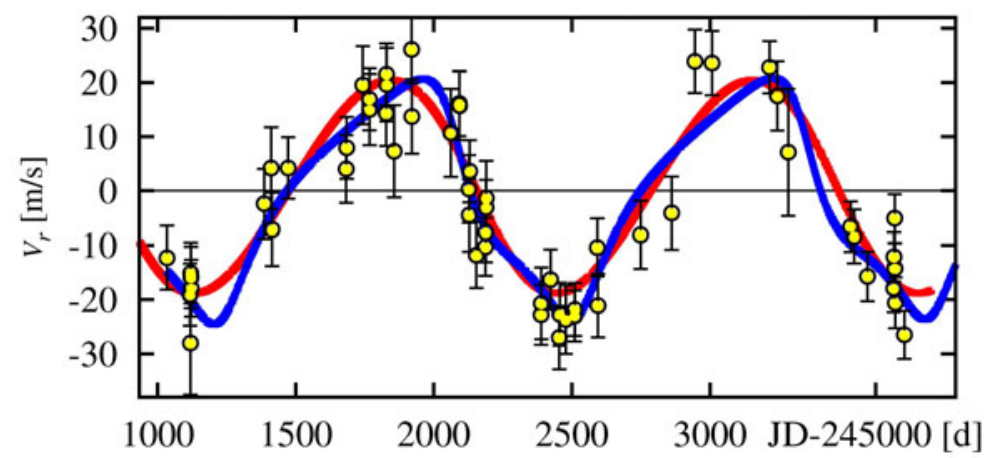

Figure 6. The RV data of $\tau^{1}$ Gruis and the synthetic signals. The red curve is for the the best, single-planet Keplerian fit yielding $\left(\chi_{\nu}^{2}\right)^{1 / 2} \sim 1$ and an $\mathrm{rms} \sim 6.1 \mathrm{~m} / \mathrm{s}$. The blue curve (darker one) is for coplanar, edge-on configuration (an rms $\sim 5.9 \mathrm{~m} / \mathrm{s}$ ) involved in 1b:1c MMR (see the top-left panel in Fig. 5 for the dynamical map).
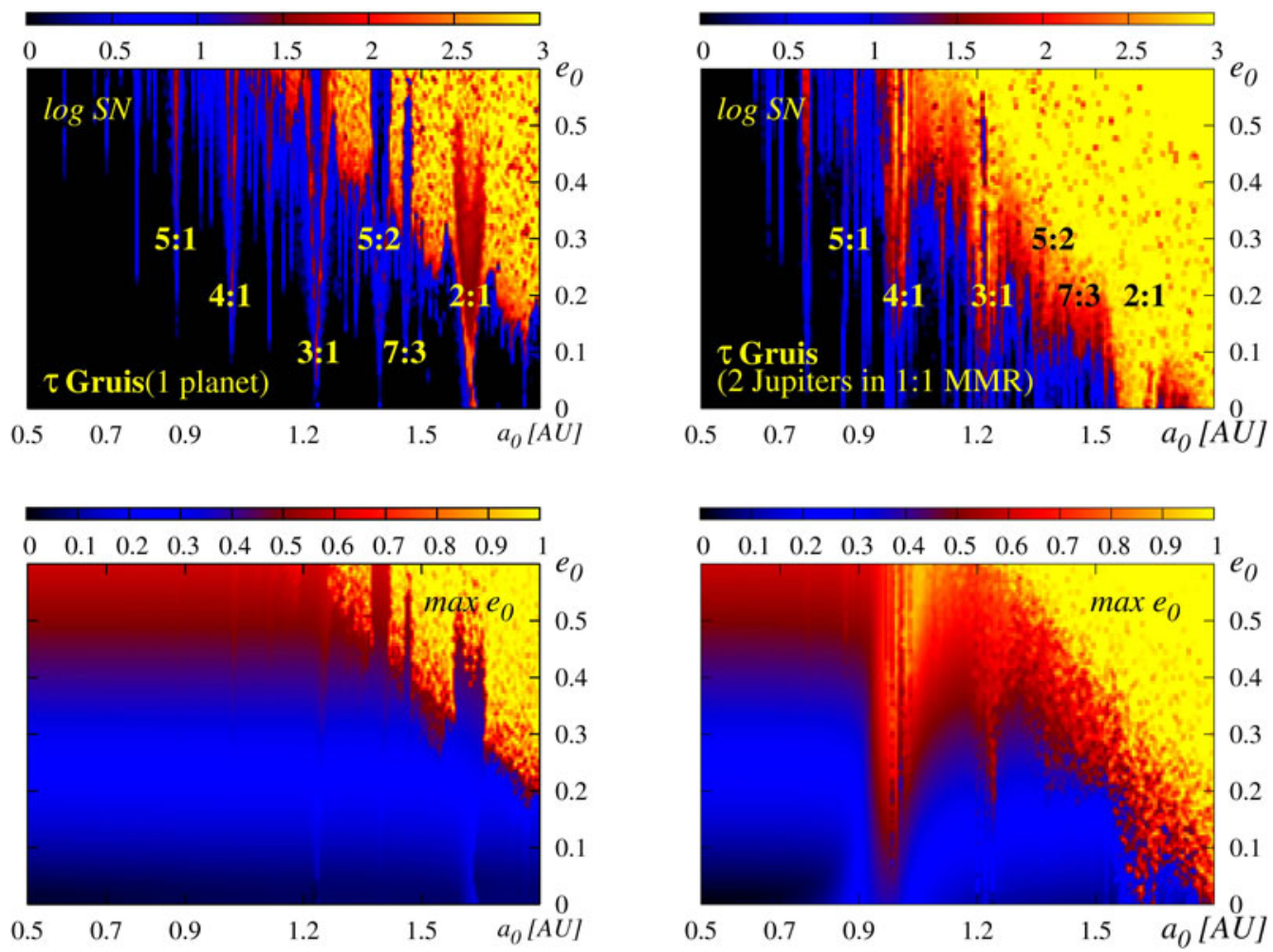

Figure 7. Dynamical maps for Earth-mass planets in the coplanar $\tau^{1}$ Gruis system. The left column is for the stability map for the best fit configuration with one Jovian planet in quasicircular orbit $(a \sim 2.5 \mathrm{AU})$, the right column is for the systems with Trojans (Table 1, fit III). Panels in the top row are for the stability indicator $\log S N$. Panels in the bottom row are for the max $e$ indicator (the integration period $\sim 50,000 \mathrm{yr}$ ). Some MMRs between the Earth-like planet and the Jovian companions are labeled. 


\begin{tabular}{|c|c|c|c|c|c|c|}
\hline \multirow[b]{2}{*}{ Parameter } & \multicolumn{2}{|c|}{ HD 155358 (fit I) } & \multicolumn{2}{|c|}{ HD 155358 (fit II) } & \multicolumn{2}{|c|}{$\tau^{1}$ Gruis (fit III) } \\
\hline & planet $\mathbf{b}$ & planet $\mathbf{c}$ & planet $\mathbf{b}$ & planet $\mathbf{c}$ & planet $\mathbf{b}$ & planet $\mathbf{c}$ \\
\hline$m \sin i\left[\mathrm{~m}_{\mathrm{J}}\right]$ & 0.827 & 0.490 & 0.863 & 0.497 & 0.401 & 0.923 \\
\hline$a[\mathrm{AU}]$ & 0.623 & 0.875 & 0.628 & 1.212 & 2.471 & 2.565 \\
\hline$e$ & 0.121 & 0.743 & 0.128 & 0.198 & 0.027 & 0.053 \\
\hline$\omega[\mathrm{deg}]$ & 131.6 & 86.11 & 161.9 & 272.0 & 99.9 & 163.8 \\
\hline $\mathcal{M}\left(t_{0}\right)[\mathrm{deg}]$ & 106.32 & 313.7 & 130.3 & 198.8 & 3.5 & 3.6 \\
\hline$\left(\chi_{\nu}^{2}\right)^{1 / 2}$ & \multicolumn{2}{|c|}{1.14} & \multicolumn{2}{|c|}{1.08} & \multicolumn{2}{|c|}{1.12} \\
\hline$\sigma_{\mathrm{j}}[\mathrm{m} / \mathrm{s}]$ & \multicolumn{2}{|c|}{5} & \multicolumn{2}{|c|}{5} & \multicolumn{2}{|c|}{4} \\
\hline $\mathrm{rms}\left[\mathrm{m} \mathrm{s}^{-1}\right]$ & \multirow{2}{*}{\multicolumn{2}{|c|}{6.31}} & \multicolumn{2}{|c|}{5.98} & \multicolumn{2}{|c|}{5.96} \\
\hline$V_{0}\left[\mathrm{~m} \mathrm{~s}^{-1}\right]$ & & & \multicolumn{2}{|c|}{10.69} & \multicolumn{2}{|c|}{-0.04} \\
\hline$M_{\star}\left[M_{\circ}\right]$ & \multicolumn{2}{|c|}{$\begin{array}{c}12.69 \\
0.87\end{array}$} & \multicolumn{2}{|c|}{0.87} & \multicolumn{2}{|c|}{1.25} \\
\hline
\end{tabular}

Table 1. The best-fit astro-centric, osculating Keplerian elements of stable, coplanar and edge-on planetary configurations at the epoch of the respective first observation. Original errors of the data are rescaled by adding the "jitter" $\sigma_{\mathrm{j}}$ in quadrature.

Moreover, our fitting method, used mainly for RV data, is quite general and may be applied to other types of observations as well. As the stability criterion, one can use the maximal Lyapunov exponent, the most stringent and formal characteristic of stable/unstable motion. Other suitable indicators like the maximal eccentricity, the spectral number, or the diffusion rate of fundamental frequencies may also be applied. These fast indicators help us to search for and find long-term stable solutions, but also make it possible to efficiently explore and to visualize the sophisticated and varying structure of the phase space. We can see the planetary system in its dynamical environment.

Many multi-planet systems are found on the edge of long-term dynamical stability. It is not clear yet whether this is a general property of multi-planet systems, the outcome of poor statistics or just the consequence of a bad choice of the RV model. Large eccentricities in multi-planet systems may "hide" other, unknown planets. Yet in that case, the dynamical modeling of the RV with stability constraints provides valuable information on the dynamical structure of the putative planetary configurations. Finding the best fits on the very edge of stable zones may provide good hints and motivation to look for alternate models of the RV.

\section{Acknowledgments}

K. G. thanks the organizers of the IAU 249 symposium for the invitation and great hospitality. We are grateful to the anonymous referee for comments that improved the manuscript. Many thanks to Boud Roukema for corrections of the text. This work is supported by the Polish Ministry of Science, Grant 1P03D 02129.

\section{References}

Arnold, V. I. Mathematical methods of classical mechanics. New York: Springer, 1978.

Barnes, R. \& Greenberg, R. ApJL, 665:L67-L70, 2007.

Baluev, R. V. arXiv:0712.3862, 2007.

Beaugé, C., Michtchenko, T. A., \& Ferraz-Mello S. MNRAS, 365:1160-1170, 2006.

Beaugé S., Sándor, Zs., Érdi, B., \& Süli, Á. A\&A, 463:359-367, 2007.

Benettin, G., Galgani, L., Giorgilli, A., \& Strelcyn, J.-M. Meccanica, pages 9-30, 1980. 
Bevington, P. R. \& Robinson, D. K. Data reduction and error analysis for the physical sciences. McGraw-Hill, 2003.

Butler, R. P. et al. Catalog of Nearby Exoplanets. ApJ, 646:505-522, 2006.

Chambers, J. E. MNRAS, 304:793-799, 1999.

Charbonneau, P. ApJs, 101:309-+, 1995.

Cincotta, P. M., Giordano, C. M., \& Simó, C. Physica D, 182:151, 2003.

Cincotta, P. M. \& Simó, C. ESs, 147:205-228, 2000.

Cochran, W. D. Endl, M. R., Wittenmyer, A., \& Bean, J. L. ApJ, 665:1407-1412, 2007.

Correia, A. C. M. et al. A\&SA, 440:751-758, 2005.

Dvorak, R., Freistetter, F., \& Kurths, J. Lecture Notes in Physics, Springer Verlag, 683, 2005.

Ferraz-Mello, S., Michtchenko, T. A., \& Beaugé, C. Chaotic Worlds: from Order to Disorder in Gravitational N-Body Dynamical Systems, 255-+, 2006.

Ferraz-Mello, S., Michtchenko, T. A., \& Beaugé, C. ApJ, 621:473-481, 2005.

Ford, E. B. AJ, 129:1706-1717, 2005.

Ford, E. B., Lystad, V., \& Rasio, F. A. Nature, 434:873-876, 2005.

Ford, E. B. \& Gaudi, B. S. ApJL, 652:L137-L140, 2006.

Froeschlé, C. Celest. Mech. E Dyn. Astr., 34:95-115, December 1984.

Goździewski, K., Breiter, S., \& Borczyk, W. MNRAS, 710, 2008 (in press).

Goździewski, K. \& Konacki, M. ApJ, 647:573-586, 2006.

Goździewski, K., Maciejewski, A. J., \& Migaszewski, C. ApJ, 657:546-558, 2007.

Goździewski, K. \& Migaszewski, C. A\&A, 449:1219-1232, 2006.

Gregory, P. C. MNRAS, 374:1321-1333, 2007.

Holman, M. J. \&Murray, N. W. AJ, 112:1278+, 1996.

Ji, J. et al. ApJL, 591:L57-L60, 2003.

Jones, H. R. A. et al. MNRAS, 341:948-952, 2003.

Laskar, J. Icarus, 88:266-291, 1990.

Laskar, J. Celest. Mech. \& Dyn. Astr., 56:191-196, 1993.

Laughlin, G., Chambers, J. E, \& Fischer, D. ApJ, 579:455-467, 2002.

Laughlin, G. \& Chambers, J. E. ApJ, 551:L109-L113, 2001.

Laughlin, G. \& Chambers, J. E. AJ, 124:592-600, 2002.

Lecar, M. et al. Annual Rev. of Astron. \& Astroph., 39:581-631, 2001.

Lee, M. H., et al. ApJ, 641:1178-1187, 2006.

Lee, M. H. \& Peale, S. J. ApJ, 592:1201-1216, 2003.

Lissauer, J. J. Rev. Mod. Phys., 71(3):835-845, 1999.

Lissauer, J. J. \& Rivera, E. J. ApJ, 554:1141-1150, 2001.

Malhotra, R. In ASP Conf. Ser. 149: Solar System Formation and Evolution, pages 37+, 1998.

Marcy, G. W., et al. ApJ, 556:296-301, 2001.

Mayor, M., et al. A\&A, 2003. astro-ph/0310316.

Michtchenko, T. A. \& Ferraz-Mello, S. ApJ, 122:474-481, 2001.

Morbidelli, A. Modern celestial mechanics: aspects of Solar system dynamics. Taylor \& Francis, 2002.

Murray, N. \& Holman, M. Nature, 410:773-779, 2001.

Nesvorný, D. \& Morbidelli, A. Celest. Mech. \& Dyn. Astr., 71:243-271, 1999.

Pepe, F., et al. A\&A, 462:769-776, 2007.

Press, W. H., Teukolsky, S. A., Vetterling, W. T., \& Flannery, B. P. Numerical Recipes in C. The Art of Scientific Computing. Cambridge Univ. Press, 1992.

Rivera, E. J. \& Lissauer, J. J. ApJ, 402:558-392, 2001.

Robutel, P. \& Laskar, J. Icarus, 152:4-28, 2001.

Šidlichovský, M. \& Nesvorný, D. Celest. Mech. \& Dyn. Astr., 65:137-148, 1997.

Smart, W. M. Text-Book on Spherical Astronomy. Cambridge Univ. Press, 1949.

Szebehely, V. Celest. Mech. ES Dyn. Astr., 34:49-64, December 1984.

Tinney, C. G., et al. ApJ, 647:594-599, 2006.

Vogt, S. S., et al. ApJ, 632:638-658, 2005.

Wisdom, J. \& Holman, M. AJ, 102:1528-1538, 1991. 